\title{
A new model of emission from microquasar jets, and possible explanation to the outliers of the fundamental plane
}

\author{
Asaf Pe'er ${ }^{1}$ and Piergiorgio Casella ${ }^{2}$ \\ ${ }^{1}$ Harvard-Smithsonian Center for Astrophysics, 60 Garden Street, Cambridge, MA 02138, USA \\ email: apeer@cfa.harvard.edu \\ ${ }^{2}$ School of Physics and Astronomy, University of Southampton, \\ Southampton, Hampshire, SO17 1BJ, UK
}

\begin{abstract}
We present a new model of emission from jets in microquasars, which implements elements from the study of jets in gamma-ray bursts to these objects. By assuming that electrons are accelerated once at the base of the jet to a power law distribution above a low energy Maxwellian, and are cooled by synchrotron emission and possible adiabatic energy losses along the jet, a wealth of spectra can be obtained. We show our theoretical results which can explain some of the key observations. In particular, we show that: (I) a flat radio spectrum, as is frequently seen, is a natural outcome of the model; (II) Strong magnetic field results in a flux decay in the optical/UV band as $F_{\nu} \sim \nu^{-1 / 2}$, irrespective of many of the uncertainties of the model. (III) An increase of the magnetic field above a critical value of $\sim 10^{5} \mathrm{G}$ leads to a sharp decrease in the flux at the radio band, while the flux at higher frequencies saturates to a constant value. We conclude that scatter in the values of the magnetic field may provide a natural explanation to the observed scatter in the radio/X ray luminosity correlation seen in these objects.
\end{abstract}

Keywords. plasmas, radiation mechanisms: non-thermal, stars: winds, outflows, X-rays: binaries

\section{Introduction}

The common theoretical modeling of emission from microquasar jets is based on the seminal work by Blandford \& Königl (1979). According to this model, the flat radio spectrum is explained as due to synchrotron emission from power law distributed electrons inside a conical jet, with a decaying magnetic field, $B(r) \propto r^{-1}$. In Pe'er \& Casella (2009), we have extended this model in several ways. The two most notable extensions are: (1) We have included a low energy cutoff in the distribution of the accelerated electrons, i.e., a power law is assumed only at a limited range $\gamma_{\min } . \gamma_{\max } \cdot(2)$ We added cooling of the electrons, due to synchrotron emission and possible adiabatic energy losses, inherently into the calculations. These two additions result in numerous non-trivial spectra.

\section{Electron energy distribution inside the jet}

As the electrons propagate inside the jet they lose their energy. As was shown by Kaiser (2006) and Pe'er \& Casella (2009), the decay of the magnetic field results in two qualitatively different regimes in electron distribution: $\nmid$ Close to the base of the jet, the magnetic field is strong, resulting in an initial rapid cooling. Further along the jet, the

$\dagger$ The analysis presented here is valid for wide jets, defined by $r(x) \propto x^{a_{j e t}}$ with $a_{\text {jet }}>1 / 2$. 

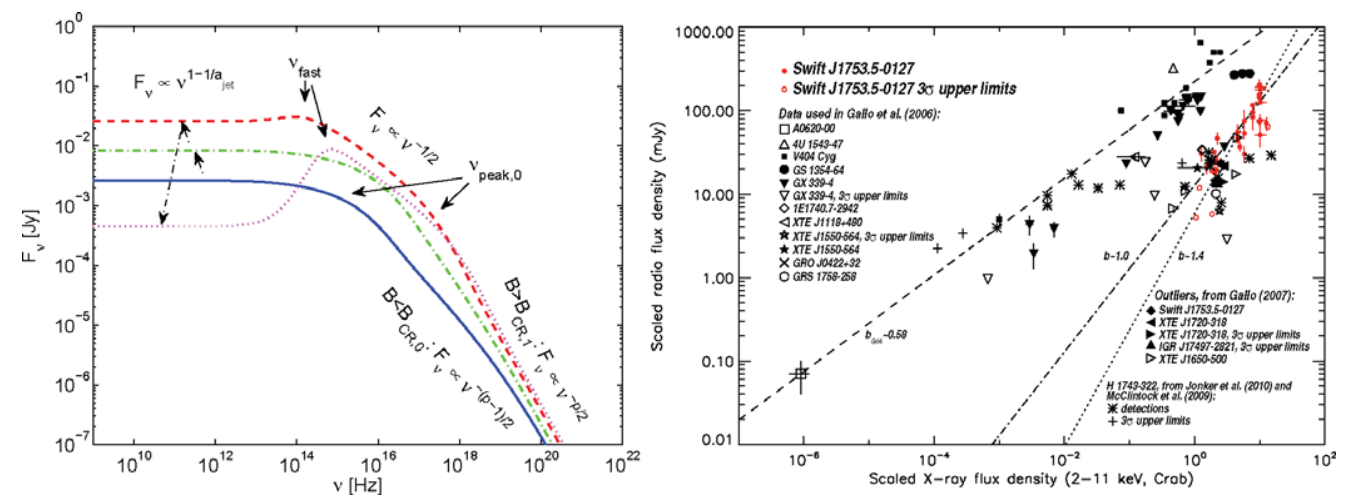

Figure 1. Left: spectrum produced for various values of the magnetic field. For high B-field, the flux at the radio frequencies is suppressed. This may provide a natural explanation to the outliers to the fundamental plane, which are shown in the right pannel (from Soleri et. al.,2010.)

magnetic field decays, and as the electrons propagate they maintain their energy, or lose it adiabatically. This fact enables to define a critical value to the magnetic field: for weak B field, $B<B_{c r, 0} \approx 10^{3} \mathrm{G}$, the electrons do not lose their energy initially, while for stronger magnetic field the initial rapid cooling is significant.

\section{The resulting spectrum}

As a wealth of spectra can be obtained, we focus here on a few key results and refer the reader to Pe'er \& Casella (2009) for further details. (1) The existence of two characteristic energies. The inclusion of a low energy cutoff in the electron distribution implies that there are two characteristic breaks in the spectrum: $\nu_{\text {peak }}$, the characteristic energy of synchrotron emission from electrons at the peak of the distribution (at $\left.\gamma_{\min }\right)$; and $\nu_{\text {break }}$, the transition between optically thin and optically thick emission. For weak magnetic field, $\nu_{\text {break }}<\nu_{\text {peak }}$. For conical jets, both frequencies decay in a similar way, resulting in a flat radio spectrum, due to the decay of $\nu_{\text {peak }}$ along the jet. (2) For high magnetic field, the electrons rapidly cool close to the jet base. In this region, the magnetic field is nearly constant, and the resulting spectrum, typically in the optic/X band is $F_{\nu} \propto \nu^{-1 / 2}$. Since the cooling occurs close to the jet base, this result is independent on the jet geometry, or on the exact nature of the particle distribution. (3) For very high magnetic field $\left(B>B_{c r, 1} \approx 10^{5} \mathrm{G}\right)$, following the rapid cooling, the peak of the emission $\left(\nu_{\text {peak }}\right)$ is below $\nu_{\text {break }}$. This results in a suppression of the radio flux, which is not accompanied by a suppression of the flux at higher frequencies, since the energetic photons are emitted close to the jet base. Casella \& Pe'er (2009) showed that this result may provide a natural explanation to the outliers often seen to the known $\mathrm{X} /$ radio flux correlation observed in the low/hard state in microquasars (the "fundamental plane").

\section{References}

Blandford, R. D. \& Königl, A. 1979, ApJ, 232, 34

Casella, P. \& Pe'er, A. 2009, ApJ, 703, L63

Kaiser, C. R. 2006, MNRAS, 367, 1083

Pe'er, A. \& Casella, P. 2009, ApJ, 699, 1919

Soleri, P., et. al. 2010, MNRAS, 406, 1471 